\title{
The effect of diabetes mellitus on evolution and outcome of tuberculosis in a prospective study
}

\author{
Alina Malic, ${ }^{*}$ Evelina Lesnic \\ Department of Pneumophthisiology, Nicolae Testemitanu State University of Medicine and Pharmacy \\ Chisinau, the Republic of Moldova
}

Authors' ORCID iDs, academic degrees and contribution are available at the end of the article

*Corresponding author - Evelina Lesnic, e-mail: evelina.lesnic@usmf.md

Manuscript received September 10, 2021; revised manuscript November 08, 2021; published online November 26,2021

\begin{abstract}
Background: In the Republic of Moldova almost $5 \%$ of the cases with tuberculosis are diagnosed annually among diabetic patients. The aim of this study was to assess the impact of diabetes mellitus on the evolution and anti-tuberculosis treatment effectiveness in a prospective study.

Material and methods: A prospective, longitudinal and case-control study, which included a total number of 252 patients diagnosed with pulmonary tuberculosis and distributed in a study group, consisting of 93 patients diagnosed with diabetes mellitus and a control group, consisting of 159 patients without glycemic disorders, was performed.

Results: This study identified that one half of the group with diabetes was detected by active screening and one third received anti-tuberculous treatment before actual episode. A similar rate of diabetic and non-diabetic patients was microbiologically positive, as well confirmed with drug-resistance. The anti-tuberculous treatment effectiveness was lower in diabetic patients, the death rate and the low treatment outcome (lost to follow-up and failed) were higher than in non-diabetic patients. The main causes of unfavorable evolution were: glycemic disorders (hyperglycemia), diabetes complications and the history of the anti-tuberculous treatment in the anamnesis.

Conclusions: The individualized approach and a tight follow-up should be performed regularly in all patients with glycemic disorders and tuberculosis for the improvement of the disease outcome.

Key words: tuberculosis, diabetes, risk factors, outcome.
\end{abstract}

Cite this article

Malic A, Lesnic E. The effect of diabetes mellitus on evolution and outcome of tuberculosis in a prospective study. Mold Med J. 2021;64(5):16-20. https:// doi.org/10.52418/moldovan-med-j.64-5.21.03.

\section{Introduction}

Diabetes mellitus (DM) is an important public health issue worldwide [1]. It is one of the four priority non-communicable diseases targeted by the sustainable development goals. Diabetes is a group of metabolic diseases characterized by hyperglycemia resulting from the disturbances in insulin secretion, insulin action or both [2]. The chronic hyperglycemia contributes to the long-term damage, dysfunction of different organs, increased risk for infections of lower respiratory tract and tuberculosis [1]. The risk for tuberculosis is increased 1.5-7.8 times in patients with uncontrolled hyperglycemia due to high levels of pro-inflammatory mediators (IL-6, TNF- $\alpha$ ) released by the infection with Mycobacterium tuberculosis in the lung tissue [3, 4].

Patients with both types of diabetes are one of the most important risk groups for tuberculosis and are exposed to the annual radiological investigation [5]. The association of diabetes and pulmonary tuberculosis is most commonly established in patients who have been diagnosed with diabetes $[6,7]$. If tuberculosis and diabetes are detected simultaneously, diabetes worsens the evolution of tuberculosis and decreases the treatment effectiveness [8-10]. The factors that contribute to the development of tuberculosis in diabetic patients are the disturbances of the innate resistance, cellular immunity, and the alveolar macrophage dysfunction [1, 3]. Late detection, the glucose metabolism disorders during the anti-tuberculous treatment, the high rate of the adverse drug reactions and acquired MDR-TB due to the low absorption of the drugs from the gastrointestinal tract contribute to the unfavorable evolution of tuberculosis $[11,12]$. Antidiabetic therapy in patients with tuberculosis should be frequently revised due to the high frequency of glycemic balance disturbances and adverse reactions to anti-tuberculous drugs. In severe forms of tuberculosis, the association of the insulin with metformin is recommended for maintaining the control of the glucose level [12]. The rate of the adverse anti-tuberculous drug effects in diabetic patients is high and ranges between $20 \%$ and $54 \%$ in MDR-TB patients [12]. The most frequent adverse drug reactions effects are: peripheral neuritis caused by Isoniazid, toxic hepatitis determined by Isoniazid, Rifampicin, Pyrazinamide, renal toxicity of Aminoglycosides (Streptomycin, Kanamycin, Amikacin) and optic neuritis due to Ethambutol [8]. In consequence, the hospitalization of the diabetic patients with tuberculosis is recommended for initiation of the antituberculous treatment [5]. 
Tuberculosis in diabetic patients is often misdiagnosed and several factors contribute: low specificity of the clinical signs and atypical localization [13]. If the relevant localization in typical tuberculosis is in the segments I, II, VI and $\mathrm{X}$, in DM patients the localization is more often in the segments III, IV and V. Also, severe, extensive destructive process is a common funding in new diagnosed cases of tuberculosis and diabetes. Despite including the patients with $\mathrm{DM}$ in the risk groups for annual screening, they are regularly detected with severe forms of tuberculosis with chronic evolution [13]. The late detection and late onset of the antituberculous therapy, dietary errors and inadequate antidiabetic treatment represent the causes of premature death in diabetic patients with TB [8-13]. Considering all the abovementioned data, the study has been performed with the aim to assess the impact of diabetes mellitus on the evolution and anti-tuberculous treatment outcome.

\section{Material and methods}

The research was prospective, selective, descriptive and cross-sectional. It included a series of 252 patients diagnosed with pulmonary tuberculosis during the period 01.01.2017-31.12.2017 in the Republic of Moldova. The inclusion criteria, which determined the selection of the patients in the research, were: age over 18 years old, TB diagnosed by a phthisiopneumologist and the signed informed consent. The cases were distributed in two groups. In the study group were selected 93 patients in which the main inclusion criteria was the DM diagnosed by an endocrinologist. In the control group were selected 159 patients without DM or history of DM. The diagnosis of pulmonary tuberculosis was confirmed through the criteria provided by the National Clinical Protocol. The sputum examination by Xpert MTB/Rif test, Ziehl-Neelsen staining, culture on Lowenstein-Jensen and liquid BACTEC media and chest $\mathrm{X}$-ray investigations were performed in all patients from both groups.

The methods used for the diagnosis of DM were: capillary glucose concentration, the fasting venous plasma glucose (FVPG) done after 10-14 hours of fast and the glycated albumin (HbA1c). The capillary glucose concentration was measured with a glucometer in a blood sample obtained from the fingertip. Glycated albumin was calculated, as the percentage of glycated albumin in the total albumin and the value $\geq 6.5 \%$ (48 $\mathrm{mmol} / \mathrm{Mol})$ and was a diagnostic indicator. The investigation schedule included the following data about the patients: biological and social peculiarities, high-risk characteristics (homelessness, migration, history of detention, the contact with a TB patient), case-management, and features of tuberculosis, glycemic indicators, anti-tuberculous treatment, antidiabetic treatment and outcome. The research was approved by the bioethics committee of Nicolae Testemitanu State University of Medicine and Pharmacy on 21st of November 2017.

The statistical analysis was performed using EpiInfo software. The data were appreciated as nominal or quan- titative. The frequency and percentage were reported for nominal data, and the mean and standard deviation were reported for continuous data. The statistical analysis of the differences between normally distributed continuous variables was tested with the t-Student test. A p-value of $<0.05$ was considered statistically significant.

\section{Results}

The distribution of the patients, according to the sex established a higher rate of men compared with the women rate in the control group (2.9) compared with the study group (2.1). The repartition of the patients, according to the age established that the young groups aged between 18 and 44 years prevailed in the control group - 100 (63\%) vs 18 (19\%) cases in the study group. A similar rate of patients aged between 45 and 54 years was determined in both groups. The patients older than 55 years statistically predominated in the study group - 53 (57\%) vs 22 (14\%) cases in the control group (tab. 1). So, young individuals aged younger 44 years, prevailed in the control group and older than 55 years in the study group. The patients' average age in the study group was $59 \pm 8$ years old, and in the control group $-38 \pm 5$ years old.

Table 1. Distribution of patients by sex and age

\begin{tabular}{|c|c|c|c|}
\hline $\begin{array}{c}\text { Sex } \\
\text { Age groups }\end{array}$ & Study group & Control group & P-value \\
\cline { 2 - 3 } & $\mathbf{N = 9 3}(\mathbf{M} \%)$ & $\mathbf{N = 1 5 9}(\mathbf{M} \%)$ & \\
\hline Men & $63(67)^{*}$ & $118(74)^{*}$ & $>0.05$ \\
\hline Women & $30(33)$ & $41(26)$ & $>0.05$ \\
\hline $18-24$ years & 0 & $20(13)$ & $<\mathbf{0 . 0 0 1}$ \\
\hline $25-34$ years & $8(8)$ & $40(25)$ & $<\mathbf{0 . 0 0 1}$ \\
\hline $35-44$ years & $10(11)$ & $40(25)$ & $<\mathbf{0 . 0 0 1}$ \\
\hline 45-54 years & $22(24)$ & $37(23)$ & $>0.05$ \\
\hline $55-64$ years & $37(40)$ & $18(11)$ & $<\mathbf{0 . 0 0 1}$ \\
\hline 65+ years & $16(17)$ & $4(3)$ & $<\mathbf{0 . 0 0 1}$ \\
\hline
\end{tabular}

The applied statistical test: the paired sample T-test, M - Mean. ${ }^{\star}$ Absolute numbers and percentages per column (in brackets).

According to the type of glycemic disorders, in the study group, the type 1 diabetes was diagnosed in 17 (18\%) patients and type 2 diabetes in $76(82 \%)$ patients, which was complicated in $39(42 \%)$ patients. The value of the FVPG exceeded the normal limit of $6 \mathrm{mmol} / \mathrm{L}$ in 68 (73\%) diabetic patients. Reporting to the total number of patients with hyperglycemia, the fasting venous plasma glucose was established in the range between 6.1 and $8 \mathrm{mmol} / \mathrm{L}$, assessed as a low increased level in 31 (46\%) patients. In 18 (26\%) cases the value ranged between 8.1 and $12 \mathrm{mmol} / \mathrm{l}$ and was assessed as middle increased value. Higher than $12 \mathrm{mmol} / \mathrm{L}$ was established in 19 (28\%) patients and was defined as uncontrolled hyperglycemia. So, in the most of the cases the fast venous plasma glucose exceeded the normal limit and in less than one half, in 39 (42\%) cases, was within the normal range being under medication control. The concentration of the glycated albumin was assessed in 68 (73\%) diabetic patients. The normal $\mathrm{HbA} 1 \mathrm{c}$ concentrations detected 
in the range between 4.8 and 5.6\%, considered as normal values, were found in 14 patients (20\% of the investigated ones). Those patients were identified with low increased hyperglycemia. The values included in the range between 6 and 6.4\% were found in 27 (40\%) patients. In 27 (40\%) cases the HbA1c concentrations exceeded $6.4 \%$ and in all cases the FVPG exceeded $12 \mathrm{mmol} / \mathrm{l}$.

The insurance provided the free of charge screening procedures. The health insurance was identified in $61(66 \%)$ cases of the study group vs 72 (45\%) cases of the control group. Homeless or people without a place of permanent residence, neither urban nor rural, predominated in the control group $39(25 \%)$ vs $4(4 \%)$ patients in the study group $(\mathrm{p}<0.05)$. A similar rate of the economic migrants, who returned from abroad in the last 12 months were identified in both groups. A couple of patients from the control group had a life history of detention. The individuals with tuberculous contact were detected in a low proportion in both groups - $5(5 \%)$ in the study group and $15(9 \%)$ in the control group. In 71 (76\%) patients of the study group were diagnosed several associated diseases, among which one was diabetes, complicated in every third case ((39 $(42 \%)$ patients). In the control group comorbidities were diagnosed in 24 (15\%) patients. So, the rate of the patients with associated diseases statistically predominated in the study group $(\mathrm{p}<0.001)$. Data are shown in the figure 1.

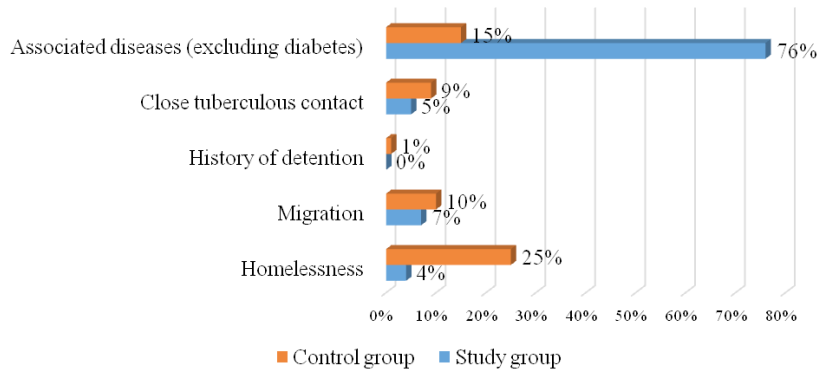

Fig. 1. Distribution according to the risk factors
The TB case-management was particularly for diabetic patients. Symptomatic screening performed by the general practitioner detected tuberculosis in a higher proportion in the control group - $68(43 \%)$ compared with the study group, $20(21 \%)$ cases. The active screening of the risk groups detected a higher proportion of the patients in the study group 49 (53\%) compared with $34(21 \%)$ cases in the control group. The rate of the patients detected through the active screening realized by the medical specialists was statistically higher in the study group 26 (28\%) compared with the control group - $10(6 \%)$. The rate of the patients detected through the symptomatic screening realized by the medical specialists was insignificantly higher in the study group 23 (25\%) compared with the control group 24 (15\%). Only patients from the control group came directly to the Pneumophthisiology hospital and avoiding the primary healthcare facilities. Thus, the specialized therapeutic intervention was more accessible for diabetic patients and the direct addressing to the pneumophthisiology hospital was preferred way for patients without glycemic disorders.

Evaluating the disease history, it was established that patients previously treated with anti-tuberculous drugs were found more often in the study group 32 (34\%) compared with $31(19 \%)$ patients in the control group. The major causes of the treatment interruption in the study group were the low treatment clinical tolerance and in the control group the treatment incompliance. The assessment of the laboratory results identified a similar rate of the patients that were positive on Ziehl-Neelson staining and conventional cultures in both groups. The drug-resistant TB was diagnosed through Xpert MTB/RIF assay in 20 (23\%) cases in the study group and $44(28 \%)$ cases in the control group and was confirmed through the conventional cultures (tab. 2).

The distribution of the patients according to the clinical and radiological criteria resulted in similar rates of the patients diagnosed with pulmonary infiltrative tuberculosis and the predomination of the fibro-cavernous form in the study group. All patients with chronic evolution had been

Table 2. Methods of detection, case-types and microbiological features

\begin{tabular}{|c|c|c|c|c|}
\hline \multirow{2}{*}{ Reference } & \multirow{2}{*}{ Case characteristics } & \multirow{2}{*}{$\begin{array}{c}\text { Study group } \\
\mathrm{N}=93(\mathrm{P} \%) \\
\end{array}$} & \multirow{2}{*}{$\begin{array}{c}\text { Control group } \\
\mathrm{N}=159(\mathrm{P} \%) \\
\end{array}$} & \multirow{2}{*}{ P-value } \\
\hline & & & & \\
\hline \multirow[t]{2}{*}{ Primary health care providers } & Detected by GPs - symptomatic patients & $20(21)^{*}$ & $68(43)^{*}$ & $<0.001$ \\
\hline & Detected by GPs - screening of HRG & $23(25)$ & $24(15)$ & $>0.05$ \\
\hline \multirow[t]{3}{*}{ Specialized health care level } & Detected by SP - symptomatic patients & $24(26)$ & $35(22)$ & $>0.05$ \\
\hline & Detected by SP - screening of HRG & $26(28)$ & $10(6)$ & $<0.001$ \\
\hline & Addressed directly to the specialized TB hospital & 0 & $22(14)$ & $<0.001$ \\
\hline \multirow[t]{2}{*}{ Case type } & New cases & $61(66)$ & $128(80)$ & $<0.001$ \\
\hline & Previously treated for TB & $32(34)$ & $31(19)$ & $<0.001$ \\
\hline \multirow[t]{3}{*}{ Microbiological results } & Positive on AFB microscopy & $38(44)$ & $79(50)$ & $>0.05$ \\
\hline & Positive on conventional cultures & $46(53)$ & $94(59)$ & $>0.05$ \\
\hline & MDR-TB & $20(23)$ & $44(28)$ & $>0.05$ \\
\hline \multirow[t]{3}{*}{ Clinical-radiological forms } & Infiltrative tuberculosis & $85(91)^{*}$ & $144(90)^{*}$ & $>0.05$ \\
\hline & Disseminated tuberculosis & $1(1)$ & $14(9)$ & $<0.001$ \\
\hline & Fibro-cavernous tuberculosis & $7(8)$ & $1(1)$ & $<0.001$ \\
\hline
\end{tabular}

The applied statistical test: the paired sample T-test, GP - general practitioner, SP - specialist, HRG - high risk group. ${ }^{\star}$ Absolute numbers and percentages per column (in brackets) 
treated with anti-tuberculous drugs, before actual episode was diagnosed.

The standard treatment for drug-susceptible TB, according to WHO recommendations is recommended in the Republic of Moldova since 2000 and all selected patients were treated according to it. The treatment for the drug-susceptible tuberculosis was administrated in 73 (78\%) cases of the study group and in $115(72 \%)$ cases of the control group. The treatment for drug-resistant tuberculosis was provided in $20(23 \%)$ patients of the study group and in 44 (28\%) patients of the control group. The treatment success rate, which included cured and those who completed the treatment was statistically higher in the control group - 140 (88\%) compared with 66 (71\%) patients in the study group. The death rate was statistically higher in the study group - $14(15 \%)$ compared with 1 (1\%) in the control group. A similar rate of patients in both groups was lost to follow-up or failed the treatment (fig. 2).

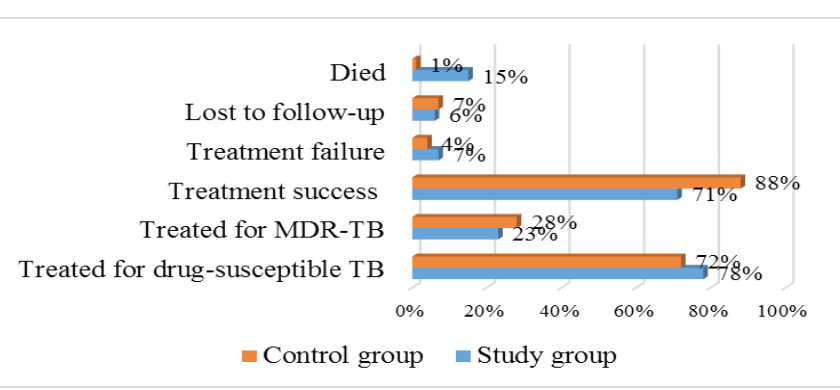

Fig. 2. Distribution according to the risk factors

\section{Discussion}

This research established that type 2 diabetes was diagnosed more often, even if the patients with type 1 diabetes were more vulnerable to infection and the occurrence of tuberculosis. The research established that tuberculosis appeared in diabetic patients with increased glycaemia and with associated complications. Similar results confirmed that uncontrolled blood glucose level increases the risk for the occurrence of tuberculosis, as the consequence of the immune disturbances $[8-10,12]$. Studies showed that men are significantly more at risk for contracting and dying from tuberculosis than women, as peculiarity of the high burden country [6]. The conducted research established a similar distribution of the diabetic patients in groups according to the sex, which demonstrated the impact of the immune disturbances on the risk for acquiring tuberculosis, more than the social risk factors. Most of the studies carried out in high tuberculosis burden countries demonstrated a high prevalence of tuberculosis in young age groups [14]. This research concluded that diabetic patients are often older 55 years which constituted a risk for acquiring type 2 diabetes. This study and the several cohort studies as well established that the social and economic vulnerability constituted a risk factor for tuberculosis in patients with DM [8-10, 12]. As a result, the research established a high rate of the diabetic patients with free access to the screening procedures, due to specific conditions, like having been retired or disabled, which is characteristic for our country. The low rate of tuberculosis detected within the investigation of the tuberculous clusters in diabetic and non-diabetic groups, reflected poor quality of the contacts' examination done in the frame of the primary health care sector. It was confirmed that the annual radiologic investigation of diabetic patients was an efficient way for tuberculosis detection in the Republic of Moldova, as being recommended by the National Clinical Protocol. The result has been sustained by another study [11]. In case the active radiological screening was routinely used, the tuberculosis with chronic evolution, such as fibrocavernous type was more often diagnosed in diabetic patients. The literature explained the effect of the uncontrolled glucose level in the blood on the releasing of the pro-inflammatory cytokines and occurrence of fibrotic changes in the lung tissue with chronic inflammation $[9,14]$. Furthermore, in the reported research the rate of previously treated for tuberculosis patients was high, which explained the fibrotic changes of the lung parenchyma in diabetic patients with long-lasting disease. A high rate of the tuberculosis recurrence and lost to follow-up among diabetic patients were shown by other researches [8-11]. The main causes contributing to the recurrence or relapse of tuberculosis in diabetic patients are: short duration of the standardized anti-tuberculous treatment, high rate of MDR-TB and adverse drug reactions, detection at the late stage, associated complications and dysfunction of the immunity [3, 4, 8-14].

The standard treatment for drug-susceptible and MDRTB was applied in a similar proportion in both groups. However, there are studies demonstrating a higher rate of drug resistance in diabetic patients $[14,15]$. The Republic of Moldova ranks in the list of the countries with a high prevalence of drug resistance, which explained that every fourth patient in both selected groups was confirmed by culture with MDR-TB [6]. The anti-tuberculous treatment effectiveness was considerably lower in the diabetic patients. The mortality rate was higher in diabetic patients, compared with non-diabetic patients, which was confirmed by other studies $[3,4,8-10,12-16]$. This study is the first to identify the peculiarities of the patients diagnosed with tuberculosis and diabetes in the Republic of Moldova and to clarify the evolution and outcome of the patients with both diseases. The limitations of this study should be also considered, due to a small number of patients included. Further studies on a larger scale are needed to establish the risk factors of both diseases.

\section{Conclusions}

The main peculiarities of diabetic patients with tuberculosis are: the average age $59 \pm 8$ years old, social-economic vulnerability, diagnosis of type 2 diabetes, which was complicated in every third case and high level of blood glucose. The present study identified that one half of the diabetic group was detected by active screening and one third received anti-tuberculous treatment before actual episode. A 
similar rate of diabetic and non-diabetic patients was microbiologically positive, as well confirmed with drug resistance. More frequently diabetic patients have been diagnosed with fibro-cavernous tuberculosis. The anti-tuberculous treatment effectiveness was lower in diabetic patients, the death rate and the low treatment outcome (lost to follow-up and failed) were higher than in non-diabetic patients. The main causes of unfavorable evolution were: glycemic disorders (hyperglycemia), diabetes complications and the history of the anti-tuberculous treatment in the anamnesis. So, the individualized approach and a tight follow-up should be performed regularly in all patients with glycemic disorders and tuberculosis for the improvement of the disease outcome.

\section{References}

1. American Diabetes Association. Standards of medical care in diabetes. Diabetes Care. 2018 Jan;41(Suppl 1):S73-S85. doi: 10.2337/dc18-S008.

2. United Nations Department of Global Communications. Sustainable Development Goals [Internet]. [Cited 2021 Aug 12]. Available from: https:// www.un.org/sustainabledevelopment/wp-content/uploads/2019/01/ SDG_Guidelines_January_2019.pdf.

3. Yorke E, Atiase Y, Akpalu J, Sarfo-Kantanka O, Boima V, Dey ID. The bidirectional relationship between tuberculosis and diabetes. Tuberc Res Treat. 2017;2017:1702578. doi: 10.1155/2017/1702578.

4. Restrepo BI, Fisher-Hoch SP, Pino PA, Salinas A, Rahbar MH, Mora F, et al. Tuberculosis in poorly controlled type 2 diabetes: altered cytokine expression in peripheral white blood cells. Clin Infect Dis. 2008;47(5):634-641. doi: 10.1086/590565.

5. Ministerul Sănătății al Republicii Moldova [Ministry of Health of the Republic of Moldova]. Tuberculoza la adulți: Protocol clinic național. [Tuberculosis in adults: Clinical national protocol] [Internet]. Chisinau: The Ministry; 2015. Romanian [cited 2021 Aug 12]. Available from: http://89.32.227.76/_files/15189-tuberculoza\%2520la\%2520adult.pdf

6. World Health Organization. Global tuberculosis report 2018 [Internet]. Geneva: WHO; 2018 [cited 2021 Aug 12]. Available from: https://apps. who.int/iris/handle/10665/274453

7. Centrul National de Management in Sanatate al Republicii Moldova [National Center for Health Management of the Republic of Moldova]. Indicatori preliminari privind sănătatea populației şi activitatea instituțiilor medico-sanitare pe anii 2016-2017 [Preliminary indices on the health of the population and activity of the medical sanitary institutions for 2016-2017]. Chisinau: The Center; 2018 [cited 2021 Aug 12]. Available from: http://old2.ms.gov.md/?q=rapoarte/indicatori-preliminariformat-prescurtat-privind-sanatatea-populatiei-si-activitatea

8. Workneh MH, Bjune GA, Yimer SA. Prevalence and associated factors of tuberculosis and diabetes mellitus comorbidity: a systematic review. PLoS One. 2017;12(4):e0175925. doi: 10.1371/journal.pone.0175925.

9. Chang JT, Dou HY, Yen CL, Wu YH, Huang RM, Lin HJ, et al. Effect of type 2 diabetes mellitus on the clinical severity and treatment outcome in patients with pulmonary tuberculosis: a potential role in the emergence of multidrug-resistance. J Formos Med Assoc. 2011;110(6):372-381. doi: 10.1016/S0929-6646(11)60055-7.

10. Siddiqui AN, Khayyam KU, Sharma M. Effect of diabetes mellitus on tuberculosis treatment outcome and adverse reactions in patients receiving directly observed treatment strategy in India: a prospective study. Biomed Res Int. 2016;2016:7273935. doi: 10.1155/2016/7273935.

11. Lesnic E, Todoriko L, Malic A, Tafuni O. Impact of diabetes comorbidity on outcome of pulmonary tuberculosis. Tuberkulez, legenevi hvorobi, VIL-infectsiia [Tuberculosis, Lung Diseases, HIV Infection] (Kiev) 2019;2(37):10-16. doi: https://doi.org/10.30978/TB2019-2-10.

12. John NM, George JM, Poornachary NM. Effect of anti-tubercular drugs and impact of glycemic status in patients with tuberculosis and concomitant diabetic-tuberculosis patients. Ind J Pharm Pract. 2017;10(2):125128. doi: 10.5530/ijopp.10.2.25.

13. Lesnic E, Malic A, Lupu E. Aportul screening-ului în depistarea tuberculozei la pacienții cu diabet zaharat [The impact of the screening in the detection of tuberculosis in patients with diabetes mellitus]. Sănătate Publică, Economie și Management în Medicină [Public Health Econ Manag Med] (Chisinau). 2018;1-2(75-76):29-33 [cited 2021 Aug 12]. Available from: http://revistaspemm.md/wp-content/ uploads/2019/05/1-275-762018.pdf

14. Harries AD, Kumar AM, Satyanarayana S, Lin Y, Zachariah R, Lönnroth $\mathrm{K}$, et al. Diabetes mellitus and tuberculosis: programmatic management issues. Int J Tuberc Lung Dis. 2015;19(8):879-886. doi: 10.5588/ ijtld.15.0069.

15. Lesnic E, Malic A, Kulcitkaia S, Niguleanu R, Jucov A, Gutu-Grecu $\mathrm{M}$, et al. The predictors of pulmonary tuberculosis in Xpert MBT/Rif positive and resistant assay patients with diabetes mellitus. Mold Med J. 2018;61(2):23-29. doi: 10.5281/zenodo.1299008.

16. Shewade HD, Jeyashree K, Mahajan P, Shah AN, Kirubakaran R, Rao $\mathrm{R}$, et al. Effect of glycemic control and type of diabetes treatment on unsuccessful TB treatment outcomes among people with TB-Diabetes: a systematic review. PLoS One. 2017;12(10):e0186697. doi: 10.1371/ journal.pone.0186697.

\section{Authors' ORCID iDs and academic degrees \\ Alina Malic, MD, PhD, Assistant Professor - https://orcid.org/0000-0002-5216-6470 \\ Evelina Lesnic, MD, PhD, Associate Professor - https://orcid.org/0000-0002-4259-0227}

\section{Authors' contribution}

AM collected the data; EL designed the research, reviewed the statistics and interpreted the data, drafted the manuscript. All the authors revised and approved the final version of the manuscript.

\section{Funding}

The study was supported by Nicolae Testemitanu State University of Medicine and Pharmacy. The authors are independent and take responsibility for the integrity of the data and accuracy of the data analysis.

\section{Ethics approval}

This study was approved by the Research Ethics Committee of Nicolae Testemitanu State University of Medicine and Pharmacy, Chisinau, the Republic of Moldova (No 44 of 26.05.2025).

\section{Conflict of interests}

No competing interests were disclosed. 